\title{
Nitrofurantoin-Associated Lung and Liver Toxicity Leading to Liver Transplantation in a Middle-Aged Patient
}

\author{
Tony K L Kiang, Jo-Ann Ford, Eric M Yoshida, and Nilufar Partovi
}

\section{INTRODUCTION}

$\mathrm{N}$ itrofurantoin is indicated for the treatment or prophylaxis of uncomplicated urinary tract infections. Currently, it is considered as second-line treatment for this type of infection because of emerging bacterial resistance. ${ }^{1}$ Nitrofurantoin is bioactivated to various reactive intermediates that interfere with bacterial ribosomal proteins and inhibit the synthesis of critical cellular macromolecules (e.g., DNA). ${ }^{2}$ Nitrofurantoin is extensively absorbed by the gastrointestinal tract, is rapidly eliminated (with a half-life of about $0.3-1 \mathrm{~h}$ ) by the kidneys, and is concentrated in the urine. ${ }^{3}$ Typical dosages in adults with normal renal function (creatinine clearance $>60 \mathrm{~mL} / \mathrm{min}$ ) are 50-100 mg PO qid for 1 week (for treatment) or 50-100 mg PO at bedtime (for prophylaxis). ${ }^{4}$ The clearance of nitrofurantoin depends on glomerular function, so its concentration in the urine, and hence its bactericidal effects, is reduced in patients with compromised creatinine clearance., ${ }^{5,6}$

Various adverse effects are associated with nitrofurantoin. Gastrointestinal complaints are common. ${ }^{7,8}$ Very rarely, neurological, hematological, pulmonary, and hepatic reactions may occur.' Peripheral neuropathy of the sensorimotor type is a common neurological complaint. ${ }^{10}$ Acute hemolytic anemia, typically associated with deficiency of glucose-6-phosphate dehydrogenase, is the predominant hematological adverse effect. ${ }^{11}$ Most nitrofurantoin-induced pulmonary toxicity is acute and immunologic in nature, but cases of chronic pulmonary fibrosis have also been reported. ${ }^{9,11}$ Likewise, hepatotoxicity caused by nitrofurantoin is generally of acute onset, but patients may also present with chronic, active hepatitis. ${ }^{12}$

Nitrofurantoin-associated pulmonary or hepatic toxicity is rare. ${ }^{9,12}$ Even rarer are patients who present with concurrent pulmonary and hepatic complications. ${ }^{11}$ We present a case of nitrofurantoin-associated lung and liver toxicity in a 57-yearold woman who required liver transplantation. We also provide an up-to-date summary of previously published cases.

\section{CASE REPORT}

A 57-year-old woman presented to the emergency department after collapsing at home.* A week before admission, the patient complained of darkened urine, low-grade fever, and pruritis. She had reported increasing fatigue and decreasing energy level for 6 months before admission. Jaundice had developed about 2 weeks before admission. Her medical history included Crohn disease, recurrent urinary tract infection, and multiple episodes of pyelonephritis (3 occurrences in the previous year). Her surgical history included appendectomy and 3 cesarean sections. On admission, the patient was not taking any medication for Crohn disease because the most recent flare-up had been more than 30 years ago. She had been taking oral nitrofurantoin $100 \mathrm{mg}$ once or twice daily and ciprofloxacin (dose not reported) for 18 months for the management of urinary tract infections. The social history was not significant (she was not an active drinker and did not smoke), and the family history was negative for cancer and hepatitis. Interestingly, her son and daughter both had diagnosed immunodeficiency. The patient had no history of substance abuse and did not use herbal products. All other causes of liver disease were excluded through exhaustive pretransplant investigation and examination of the explanted liver by liver disease specialists.

At the time of admission, the patient appeared jaundiced and short of breath. On examination, the patient was alert and oriented. Asterixis was not evident, and no stigmata associated with liver disease were found. The results of an abdominal examination were normal, and no organomegaly or peripheral edema was evident. The liver echotexture did not have any coarseness or nodularity that would suggest cirrhosis or chronic liver disease. Computed tomography of the chest

*The patient's consent for publication was not obtained; all identifying information has been removed from the article. 
indicated interstitial lung disease with peripheral cavities. Chest radiography revealed bilateral infiltrates. Liver function tests showed elevation of aspartate aminotransferase (1444 units/L), alanine aminotransferase (1926 units/L), direct bilirubin (221 $\mu \mathrm{mol} / \mathrm{L})$, total bilirubin $(309 \mu \mathrm{mol} / \mathrm{L})$, alkaline phosphatase (169 units/L), and $\gamma$-glutamyltransferase (276 units/L). Laboratory findings included white blood cell count $7.3 \times$ $10^{9} / \mathrm{L}$, hemoglobin $140 \mathrm{~g} / \mathrm{L}$, platelets $292 \times 10^{\circ} / \mathrm{L}$, international normalized ratio 1.8 , partial thromboplastin time $36 \mathrm{~s}$, creatinine $61 \mu \mathrm{mol} / \mathrm{L}$, and albumin $26 \mathrm{~g} / \mathrm{L}$. Immunoglobulin A, immunoglobulin $M$, immunoglobulin $G, \alpha_{1}$-antitrypsin, ceruloplasmin, hepatitis B antigen, antibody to hepatitis B core antigen (anti-HBc), antibody to hepatitis $\mathrm{C}$ virus (anti-HCV), and anti-smooth muscle antibody were all negative or within normal limits. As well, serum levels of salicylate and acetaminophen were below the level of detection.

Nitrofurantoin was discontinued before admission, but the patient's liver dysfunction progressed despite oral administration of prednisone $(20 \mathrm{mg} /$ day) in hospital. Signs of hepatic encephalopathy developed, and the patient subsequently received a liver transplant, about 1 month after admission to hospital. Since the transplant, the patient's lung disease has been clinically silent, with no need for pulmonary medications, respirology consultations, or supplemental oxygen. Examination of a liver specimen showed large zones of necrotic liver, extensive bile duct proliferation, acute portal inflammation, and cholestasis. There was no evidence of malignancy or cirrhosis. At the time of writing, 2 years after the transplant, the patient was stable and had normal allograft function.

\section{DISCUSSION}

In the case reported here, concurrent interstitial lung disease and hepatotoxicity developed in a 57-year-old woman secondary to long-term use of nitrofurantoin (over 18 months). The Naranjo probability score was 3 , which indicated "possible" causality between nitrofurantoin and the development of concurrent lung and liver toxicity. ${ }^{13}$ Clinical judgement on the part of various hepatic and pulmonary specialists ultimately led to the conclusion of causality. Autoimmune hepatitis was an unlikely differential diagnosis given the normal levels of immunoglobulin $G$ and anti-smooth muscle antibody and the lack of autoimmune hepatitis features on histologic examination of the explanted liver. Ciprofloxacin, which was being taken concurrently by the patient, has also been associated with liver injury, but liver toxicity from ciprofloxacin is mainly of the cholestatic type ${ }^{14,15}$ and no case of combined lung and liver toxicity has been reported with ciprofloxacin. Accordingly, the pulmonary and hepatic manifestations observed in this patient were attributed to nitrofurantoin alone.

To summarize the published cases of nitrofurantoinassociated concurrent lung and liver toxicity, PubMed and
Embase were searched with various combinations of the following terms: nitrofurantoin, hepatotoxicity, pulmonary toxicity, liver, lung, hepatitis, liver necrosis, pulmonary fibrosis, and interstitial pneumonitis. Only articles published in English and searchable before October 2010 were included. The titles and abstracts were screened for relevance, and 9 published articles $^{16-24}$ describing 10 case reports were identified (Table 1).

To the best of our knowledge, this is the first summary incorporating all cases of nitrofurantoin-induced concurrent pulmonary and hepatic toxicity published in English. Most cases ( 9 of 10) occurred in women, most patients were generally older (in 8 of the 10 cases, the patients were older than 57 years; mean age 59 years), and most were receiving nitrofurantoin for the management of urinary tract infection. No clear dose-response relationship was evident, as the dose of nitrofurantoin ranged between 50 and $300 \mathrm{mg}$ per day, which is within the recommended range. ${ }^{4}$ The onset of symptoms could generally be described as "late" (i.e., 11 months to 11 years after initiation of therapy), and it was difficult to discern whether the pulmonary toxicity preceded the hepatotoxicity or vice versa. Common symptoms included jaundice, weight loss, malaise, dyspnea, and dry cough. Rales or crackles could be heard, usually from both lungs, and in the majority of cases, chest radiography indicated a pattern of interstitial lung disease. The results of pulmonary function tests, when available, suggested impairment of the restrictive type.

Most liver biopsies illustrated a pattern of chronic active hepatitis with piecemeal necrosis and/or infiltration by nonparenchyma cells, although normal biopsy results were also reported. Laboratory investigations often revealed increased erythrocyte sedimentation rate and markedly elevated transaminases, alkaline phosphatase, $\gamma$-glutamyltransferase, or bilirubin. Increased international normalized ratio, partial thromboplastin time, or activated partial thromboplastin time, suggesting compromised liver synthetic function, and positive antinuclear antibodies or anti-smooth muscle antibodies, suggesting immunologic involvement, were also commonly reported. Upon discontinuation of nitrofurantoin, most of the patients improved with respect to clinical status, pulmonary function, and liver function, in the absence of corticosteroid therapy. The time to recovery varied from 3 weeks to 6 months. Recovery of liver function was faster than or equal to that of pulmonary function in a few cases, ${ }^{16,19,20,24}$ but not in others. ${ }^{17,22}$ Two of the patients died of subsequent complications, despite corticosteroid therapy in one case..$^{18,21}$

The incidence of pulmonary toxicity associated with nitrofurantoin has been estimated at $0.00002 \%$ to $0.0009 \%$, ${ }^{9}$ and that of hepatotoxicity at $0.0003 \%{ }^{9}$ to $0.035 \%{ }^{12}$ The incidence of concurrent pulmonary and hepatic toxicity remains unknown, although it is probably less than the incidence of either form of toxicity alone. Reinhart and others ${ }^{17}$ estimated 


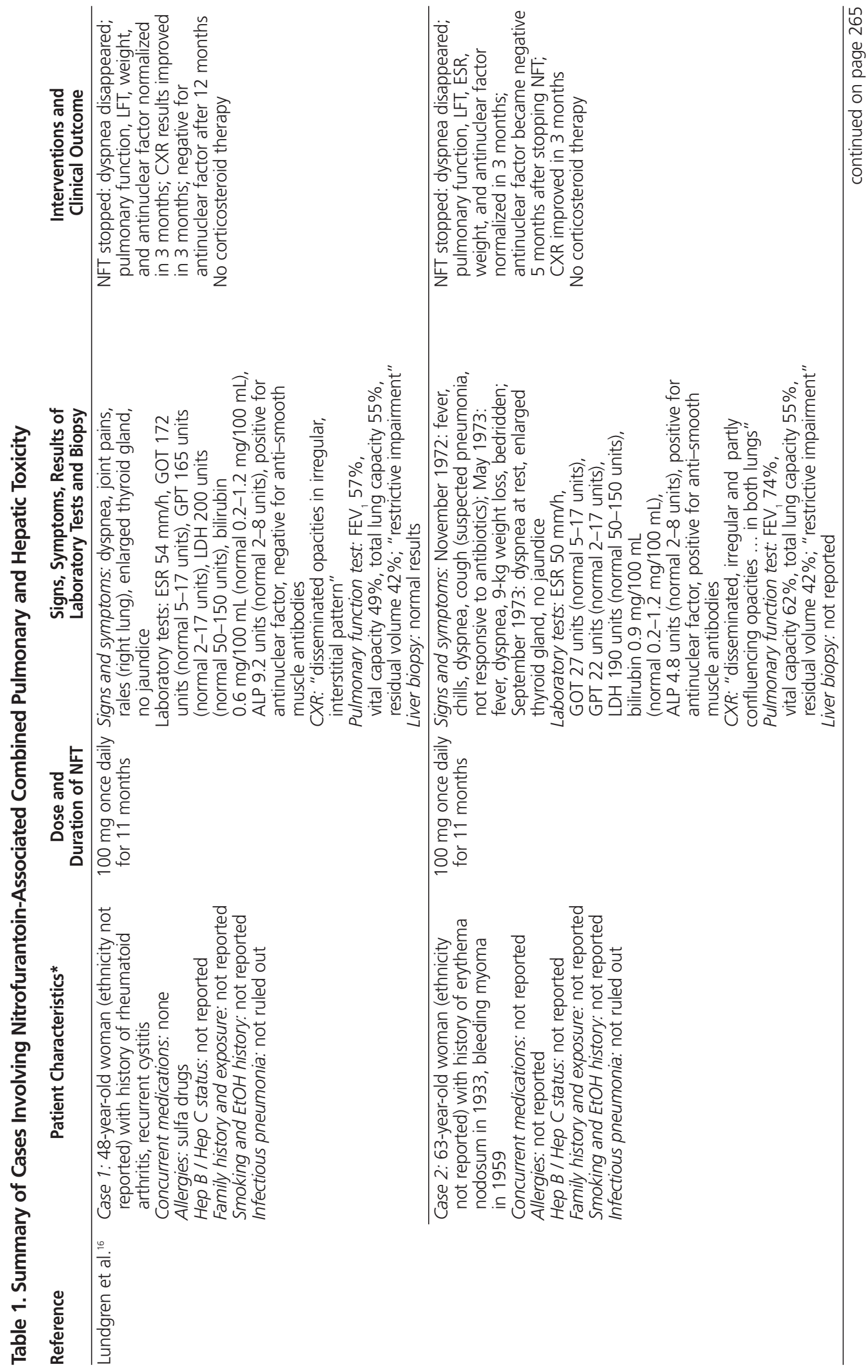


For permission to reprint multiple copies or to order presentation-ready copies for distribution, contact CJHP at cjhpedit@cshp.ca

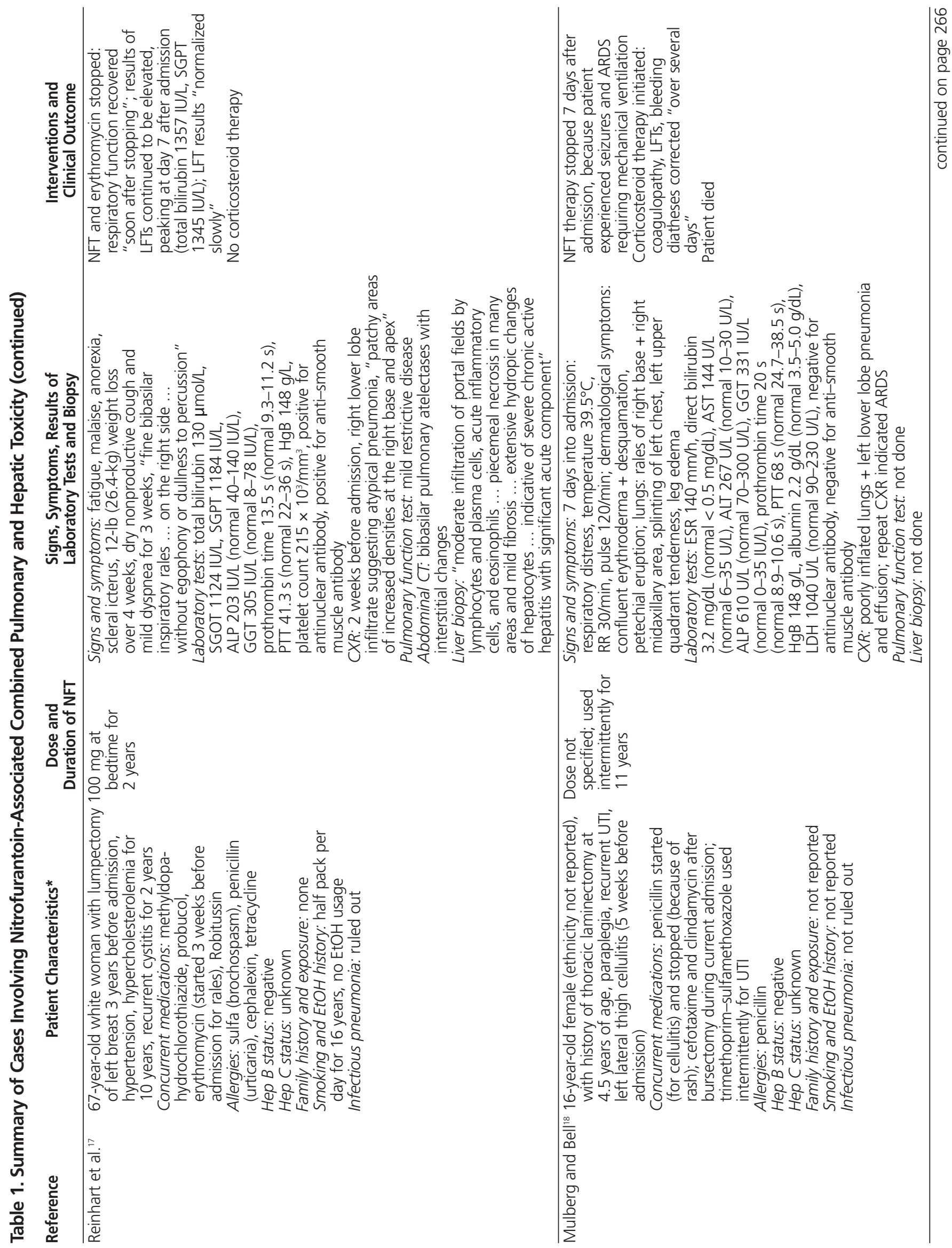


For permission to reprint multiple copies or to order presentation-ready copies for distribution, contact CJHP at cjhpedit@cshp.ca

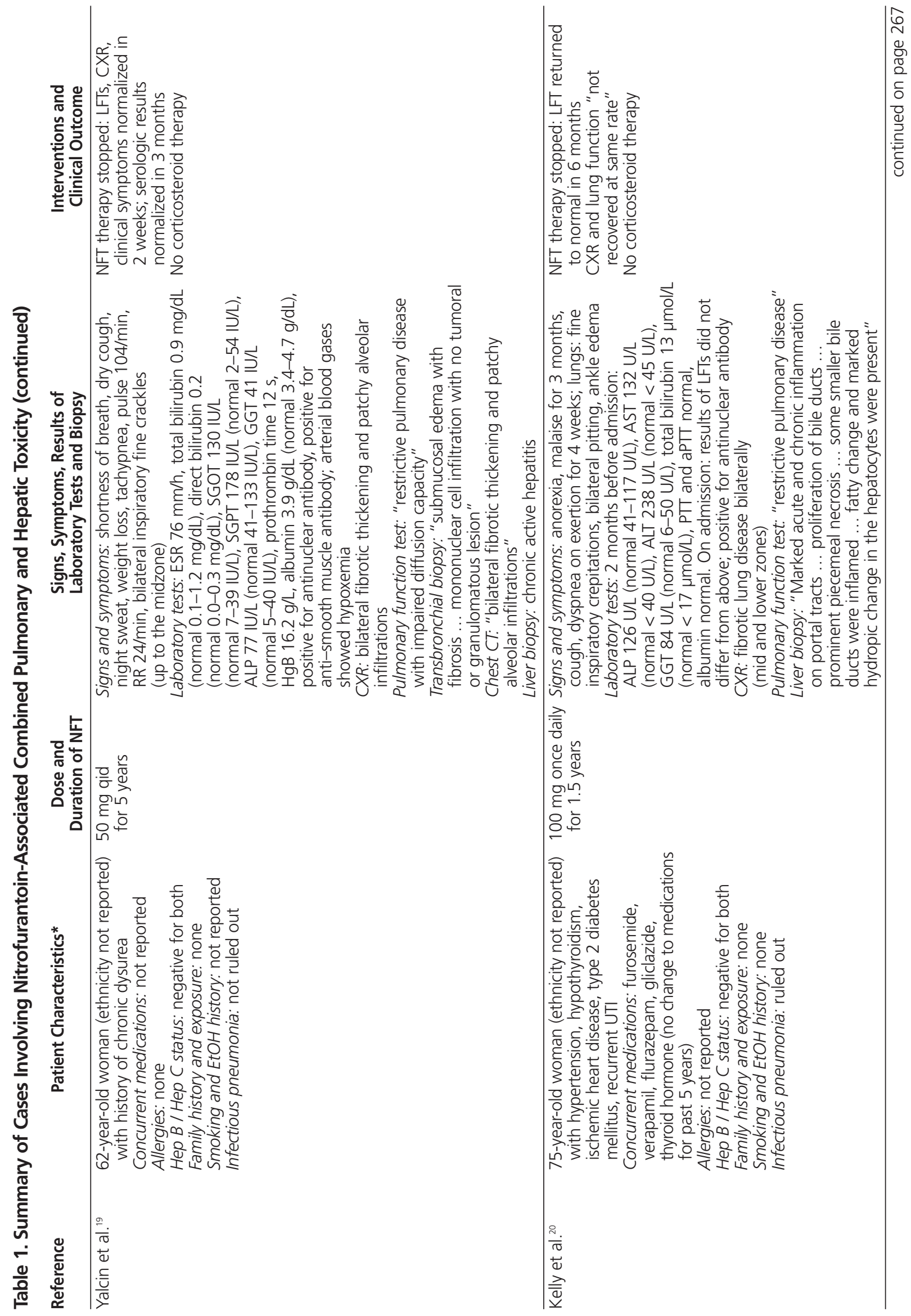


For permission to reprint multiple copies or to order presentation-ready copies for distribution, contact CJHP at cjhpedit@cshp.ca

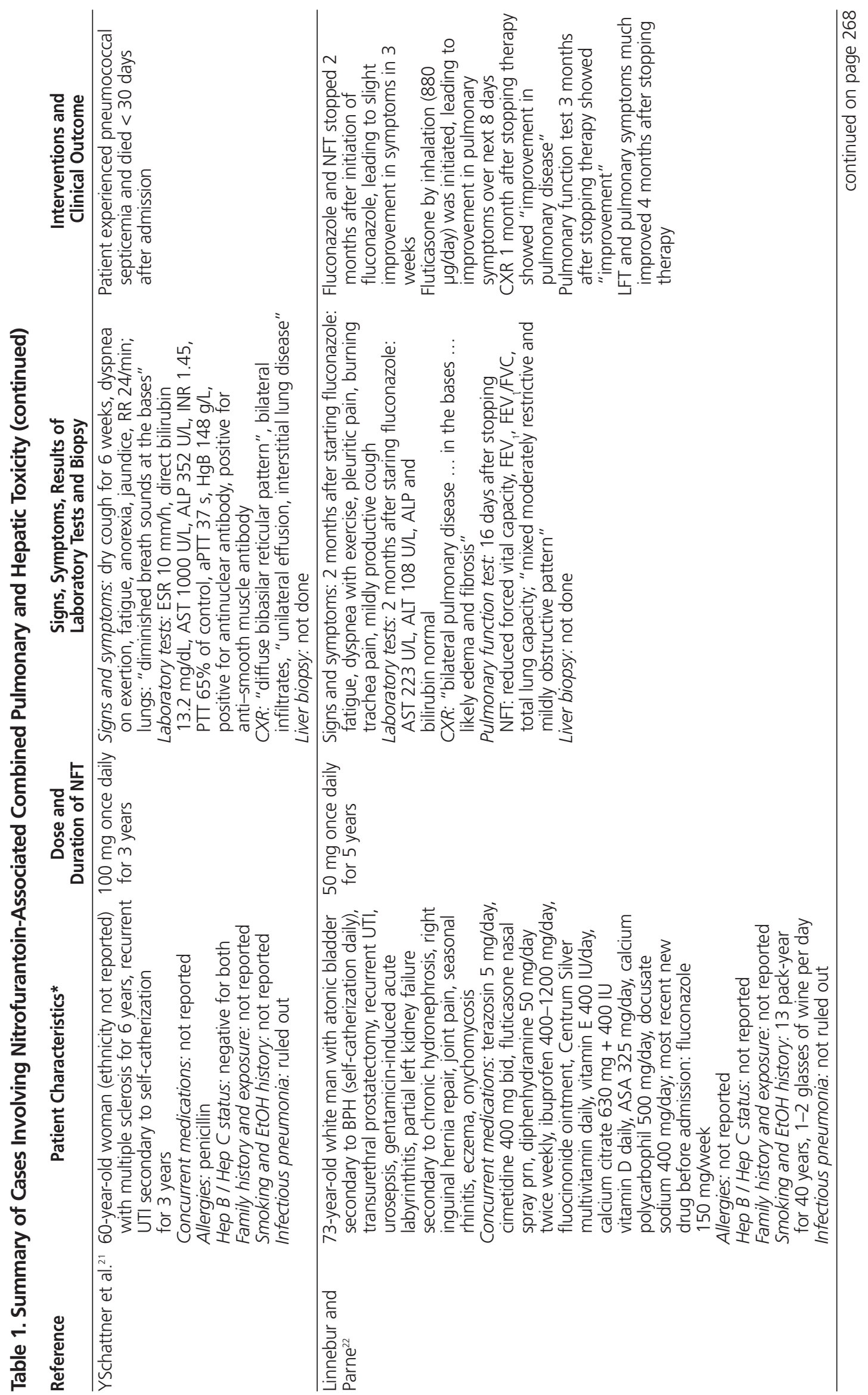




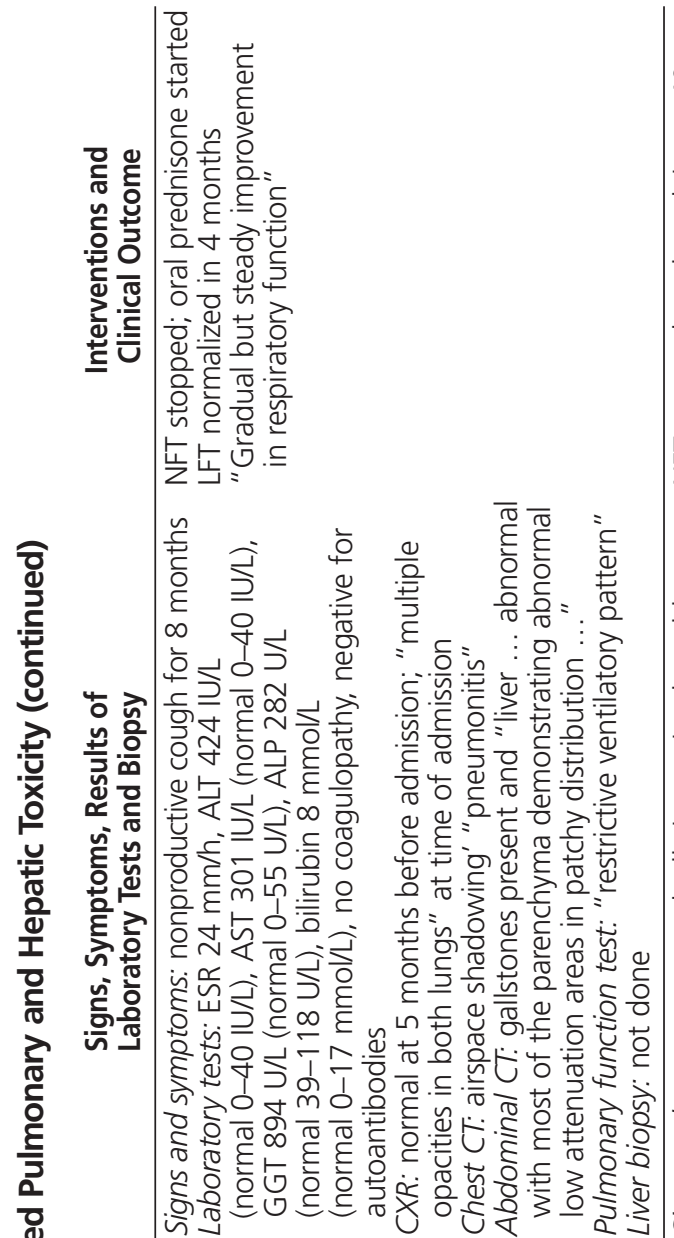

\section{$\stackrel{8}{\varepsilon}$}

市

产

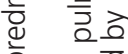

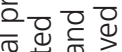

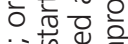

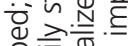

응흉 르응 을

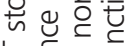

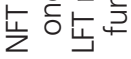

崖

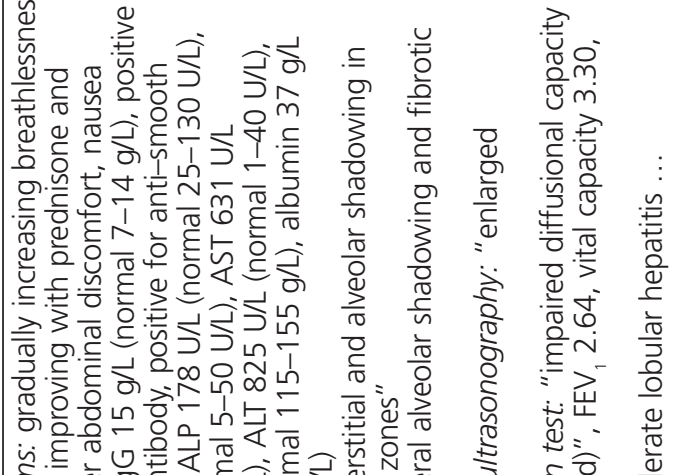

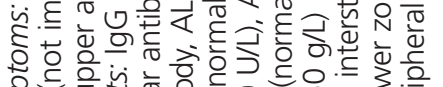

है

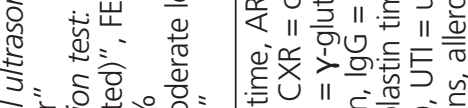

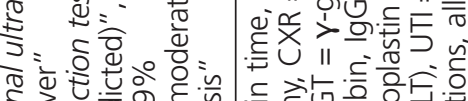

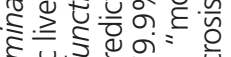

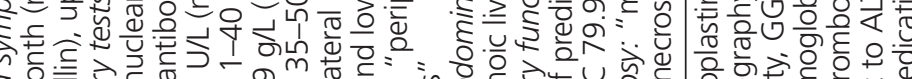

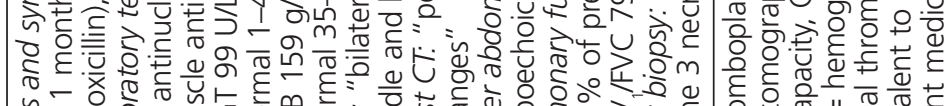

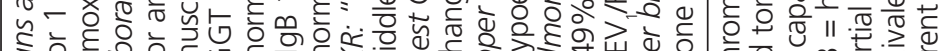

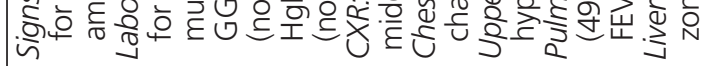

त्रेत्र

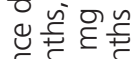

등ㅇㅇㅇํㅇ

है 6

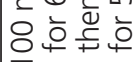

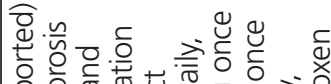

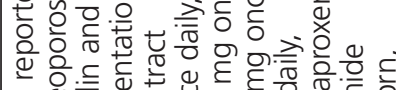

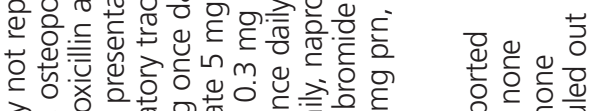

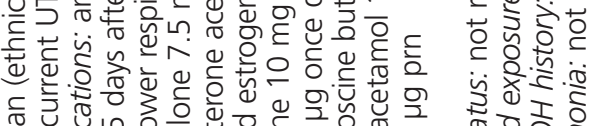

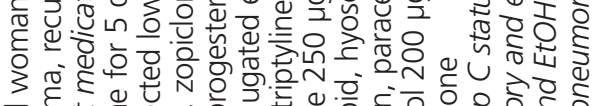

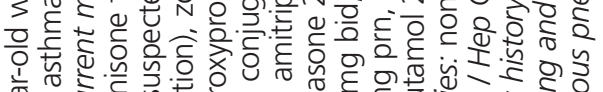

बै

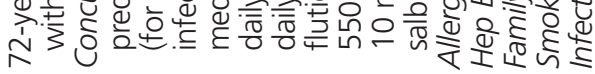

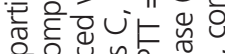

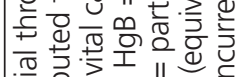

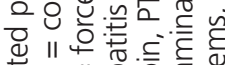

T.

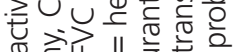

II 응

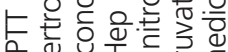

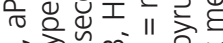

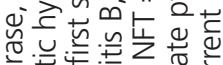

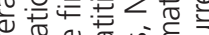

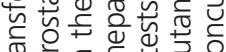

震产.

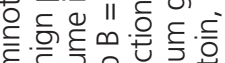

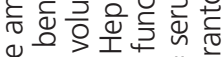

ه

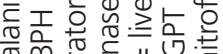

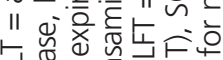

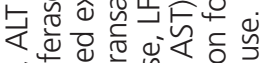

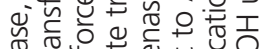

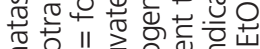

的.

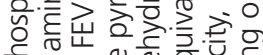

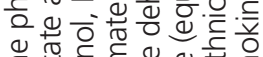

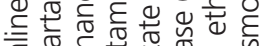

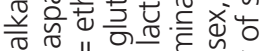

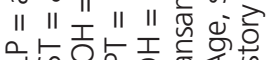


the incidence of combined toxicity at $3.9 \times 10^{-11}$ per course of therapy, which might explain the small number of cases that have reported in the literature.

According to Sovijarvi and others, ${ }^{25}$ most cases of nitrofurantoin-associated pulmonary toxicity can be classified as having acute onset, although numerous reports of chronic pulmonary toxicity have also been published. ${ }^{25-28}$ The mean delay before onset of chronic pulmonary toxicity has been reported as 30 months, ${ }^{29}$ and this condition lacks a dose-response relationship, occurs predominantly in women, and is often slow to resolve.' Common manifestations have included interstitial pneumonitis and fibrosis, which may lead to shortness of breath and cough (often dry). Chest radiography often indicates bilateral pleural effusion, with or without pulmonary infiltrates. ${ }^{30}$

Similar to lung toxicity, acute liver toxicity from nitrofurantoin is more common than chronic liver injury. ${ }^{912}$ Stricker and others ${ }^{12}$ summarized cases published before 1988, noting that chronic hepatotoxicity occurred predominantly in women, had an onset later than 6 months, and lacked a clear doseresponse relationship. Common signs or symptoms included jaundice, hepatomegaly, malaise, anorexia, weight loss, nausea, and vomiting. About $70 \%$ of patients had anti-smooth muscle antibodies, and about $80 \%$ had antinuclear factors. Liver biochemistry tests suggested hepatocellular with occasional mixed cholestatic-hepatocellular abnormalities, and liver biopsy often indicated a pattern of chronic active hepatitis. ${ }^{12}$ Only a handful of case reports of nitrofurantoin-associated hepatotoxicity have been published since $1988,{ }^{31-36}$ and the clinical picture from these more recent cases was similar to that summarized by Stricker and others. ${ }^{12}$

The mechanism or mechanisms of pulmonary and hepatic toxicity remain unknown. In vitro and animal experiments have implicated a role for bioactivation and oxidative stress in both pulmonary toxicity ${ }^{37,38}$ and hepatotoxicity, ${ }^{39.43}$ although human studies are still lacking. Positive titres of antinuclear factor and anti-smooth muscle antibodies in cases of pulmonary toxicity ${ }^{44}$ and hepatotoxicity ${ }^{12}$ also suggest an immune-mediated reaction. Cases of rapid onset after drug rechallenge $^{31,45}$ and marked sensitization ${ }^{46}$ support the immunological mechanism. From these observations, it might be hypothesized that there is more than one cause of nitrofurantoinassociated hepatotoxicity and that the immunologic injury could be the mechanism in some cases. Interestingly, signs and symptoms in cases of combined toxicity (Table 1 and the current case) corresponded to those described in cases of isolated lung or liver toxicity. This suggests that similar mechanisms are responsible for toxic effects in the individual organs, irrespective of the patient's presentation (i.e., singular organ toxicity or combined lung and liver toxicity). However, one can hypothesize that different mechanisms may be respon- sible for pulmonary and hepatic toxicity, because a common mechanism would likely yield a much higher incidence of combined toxicity, which is not the case.

The patient described here resembled patients in previously published cases (summarized in Table 1) with respect to demographic characteristics (specifically age and sex) and onset of symptoms. The clinical symptoms and laboratory findings were also comparable, except for, in the current case, the lack of elevation of markers suggestive of an immunologic reaction (e.g., antinuclear factor and anti-smooth muscle antibodies). As discussed above, the mechanisms of concurrent lung and liver toxicity could be multifactorial, and there are certainly cases in the literature that also appeared to lack an immunological component (Table 1). Our comprehensive literature summary, in conjunction with the case reported here, suggests that a distinguishable pattern of toxicity is available to aid clinicians in recognizing or possibly preventing full-blown pulmonary and hepatic toxicity from nitrofurantoin. Conversely, a clear pattern of clinical resolution (e.g., rate of recovery or responsiveness to corticosteroids) is not discernible from the small number of cases available. However, in the majority of published cases, the patients appear to have recovered, with respect to hepatic and pulmonary function, after withdrawal of nitrofurantoin and provision of supportive treatment (Table 1). The case reported here certainly supports the "reversible toxicity hypothesis" with respect to the patient's lung disease and the successful and uneventful recovery after the liver transplant.

We recommend that clinicians use the findings in this case report and literature summary to identify patients at risk of nitrofurantoin-associated lung and liver toxicity. We also recommend that patients receiving long-term nitrofurantoin therapy who are in the demographic group identified here be considered at risk of toxicity and undergo monitoring of pulmonary and hepatic function.

\section{References}

1. Stamm WE, Hooton TM. Management of urinary tract infections in adults. N Engl J Med 1993;329(18):1328-1334.

2. Guay DR. An update on the role of nitrofurans in the management of urinary tract infections. Drugs 2001;61(3):353-364.

3. Conklin JD. The pharmacokinetics of nitrofurantoin and its related bioavailability. Antibiot Chemother 1978;25:233-252.

4. Petri WA. Sulfonamides, trimethoprim, sulfamethoxazole, quinolones, and agents for urinary tract infections. In: Brunton LL, Lazo JS, Parker KL, editors. Goodman \& Gilman's the pharmacological basis of therapeutics. New York (NY): McGraw Hill; 2006. p. 1111-1125.

5. Sachs J, Geer T, Noell P, Kunin CM. Effect of renal function on urinary recovery of orally administered nitrofurantoin. $N$ Engl J Med 1968; 278(19):1032-1035.

6. Sullivan JW, Bueschen AJ, Schlegel JU. Nitrofurantoin, sulfamethizole and cephalexin urinary concentration in unequally functioning pyelonephritic kidneys. J Urol 1975;114(3):343-347.

7. Hasen HB, Moore TD. Nitrofurantoin: a study in vitro and in vivo in one hundred cases of urinary infection. J Am Med Assoc 1954;155(17): $1470-1473$. 
8. Holloway WJ. Nitrofurantoin in urinary tract infections. I. Nitrofurantoin revisited. Del Med J 1972;44(4):99-104.

9. D’Arcy PF. Nitrofurantoin. Drug Intell Clin Pharm 1985;19(7-8): 540-547.

10. Toole JF, Parrish ML. Nitrofurantoin polyneuropathy. Neurology 1973;23(5):554-559.

11. Holmberg L, Bornan G, Bottiger LE, Eriksson B, Spross R, Wessling A. Adverse reactions to nitrofurantoin. Analysis of 921 reports. Am J Med 1980;69:733-738.

12. Stricker BH, Blok AP, Claas FH, Van Parys GE, Desmet VJ. Hepatic injury associated with the use of nitrofurans: a clinicopathological study of 52 reported cases. Hepatology 1988;8(3):599-606.

13. Naranjo CA, Busto U, Sellers EM, Sandor P, Ruiz I, Roberts EA, et al. A method for estimating the probability of adverse drug reactions. Clin Pharmacol Ther 1981;30(2):239-245.

14. Cholongitas E, Georgousaki C, Spyrou S, Dasenaki M. Ciprofloxacininduced acute cholestatic hepatitis. Ann Hepatol 2009;8(4):400-401.

15. Hautekeete ML, Kockx MM, Naegels S, Holvoet JK, Hubens H, Kloppel G. Cholestatic hepatitis related to quinolones: a report of two cases. $J$ Hepatol 1995;23(6):759-760.

16. Lundgren R, Back O, Wiman LG. Pulmonary lesions and autoimmune reactions after long-term nitrofurantoin treatment. Scand J Respir Dis 1975;56(4):208-216.

17. Reinhart HH, Reinhart E, Korlipara P, Peleman R. Combined nitrofurantoin toxicity to liver and lung. Gastroenterology 1992;102(4 Pt 1):1396-1399.

18. Mulberg AE, Bell LM. Fatal cholestatic hepatitis and multisystem failure associated with nitrofurantoin. J Pediatr Gastroenterol Nutr 1993; 17(3):307-309.

19. Yalcin S, Sahin A, Yalcin B, Altinok G. Nitrofurantoin toxicity to both liver and lungs. Liver 1997;17(3):166-167.

20. Kelly BD, Heneghan MA, Bennani F, Connolly CE, O'Gorman TA. Nitrofurantoin-induced hepatotoxicity mediated by CD8+ T cells. Am J Gastroenterol 1998;93(5):819-821.

21. Schattner A, Von der Walde J, Kozak N, Sokolovskaya N, Knobler H. Nitrofurantoin-induced immune-mediated lung and liver disease. $\mathrm{Am} \mathrm{J}$ Med Sci 1999;317(5):336-340.

22. Linnebur SA, Parnes BL. Pulmonary and hepatic toxicity due to nitrofurantoin and fluconazole treatment. Ann Pharmacother 2004; 38(4):612-616.

23. Koulaouzidis A, Bhat S, Moschos J, Tan C, De Ramon A. Nitrofurantoininduced lung- and hepatotoxicity. Ann Hepatol 2007;6(2):119-121.

24. Peall AF, Hodges A. Concomitant pulmonary and hepatic toxicity secondary to nitrofurantoin: a case report. J Med Case Reports 2007;1:59.

25. Sovijarvi AR, Lernola M, Stenius B, Idanpaan-Heikkila J. Nitrofurantoininduced acute, subacute and chronic pulmonary reactions. Scand J Respir Dis 1977;58(1):41-50.

26. Hawley HB, Payne CB, Kane KK. Nitrofurantoin pneumonitis. Med Times 1982;110:34-39.

27. Ruikka I, Vaissalo T, Saarimaa H. Progressive pulmonary fibrosis during nitrofurantoin therapy. A case history with autopsy report. Scand J Respir Dis 1971;52(3):162-166.

28. Kursh ED, Mostyn EM, Persky L. Nitrofurantoin pulmonary complications. J Urol 1975;113(3):392-395.

29. Simonian SJ, Kroeker EJ, Boyd DP. Chronic interstitial pneumonitis with fibrosis after long-term therapy with nitrofurantoin. Ann Thorac Surg 1977;24(3):284-288.

30. Hailey FJ, Glascock HW, Hewitt WF. Pleuropneumonic reactions to nitrofurantoin. N Engl J Med 1969;281(20):1087-1090.

31. Paiva LA, Wright PJ, Koff RS. Long-term hepatic memory for hypersensitivity to nitrofurantoin. Am J Gastroenterol 1992;87(7):891-893.

32. Mollison LC, Angus P, Richards M, Jones RM, Ireton J. Hepatitis due to nitrofurantoin. Med J Aust 1992;156(5):347-349.

33. Burgert SJ, Burke JP, Box TD. Reversible nitrofurantoin-induced chronic active hepatitis and hepatic cirrhosis in a patient awaiting liver transplantation. Transplantation 1995;59(3):448-449.
34. Amit G, Cohen P, Ackerman Z. Nitrofurantoin-induced chronic active hepatitis. Isr Med Assoc J 2002;4(3):184-186.

35. Beigel R, Perets R, Mouallem M. Acute kidney injury, hepatitis, and CPK elevation associated with nitrofurantoin therapy. Am J Med Sci 2009;337(2):132-133.

36. Aksamija A, Horvat G, Habek D, Zalac D, Jendris E. Nitrofurantoininduced acute liver damage in pregnancy. Arh Hig Rada Toksikol 2009;60(3):357-361.

37. Suntres ZE, Shek PN. Nitrofurantoin-induced pulmonary toxicity. In vivo evidence for oxidative stress-mediated mechanisms. Biochem Pharmacol 1992;43(5):1127-1135.

38. Martin WJ, Powis GW, Kachel DL. Nitrofurantoin-stimulated oxidant production in pulmonary endothelial cells. J Lab Clin Med 1985; 105(1):23-29.

39. Silva JM, McGirr L, O’Brien PJ. Prevention of nitrofurantoin-induced cytotoxicity in isolated hepatocytes by fructose. Arch Biochem Biophys 1991;289(2):313-318.

40. Rossi L, Silva JM, McGirr LG, O'Brien PJ. Nitrofurantoin-mediated oxidative stress cytotoxicity in isolated rat hepatocytes. Biochem Pharmacol 1988;37(16):3109-3117.

41. Paterna JC, Boess F, Staubli A, Boelsterli UA. Antioxidant and cytoprotective properties of D-tagatose in cultured murine hepatocytes. Toxicol Appl Pharmacol 1998;148(1):117-125.

42. Dubuisson ML, De Wergifosse B, Kremers P, Marchand-Brynaert J, Trouet A, Rees JF. Protection against nitrofurantoin-induced oxidative stress by coelenterazine analogues and their oxidation products in rat hepatocytes. Free Radic Res 2001;34(3):285-296.

43. Klee S, Nurnberger MC, Ungemach FR. The consequences of nitrofurantoin-induced oxidative stress in isolated rat hepatocytes: evaluation of pathobiochemical alterations. Chem Biol Interact 1994;93(2):91-102.

44. Holmberg L, Boman G. Pulmonary reactions to nitrofurantoin. 447 cases reported to the Swedish Adverse Drug Reaction Committee 1966-1976. Eur J Respir Dis 1981;62(3):180-189.

45. Goldstein LI, Ishak KG, Burns W. Hepatic injury associated with nitrofurantoin therapy. Am J Dig Dis 1974;19(11):987-998.

46. Berry WR, Warren GH, Reichen J. Nitrofurantoin-induced cholestatic hepatitis from cow's milk in a teenaged boy. West J Med 1984;140(2): $278-280$

Tony K L Kiang, BSC(Pharm), PhD, ACPR, is a Clinical Pharmacist at Providence Health Care and a postdoctoral fellow (part-time) in the Faculty of Pharmaceutical Sciences, The University of British Columbia, Vancouver, British Columbia.

Jo-Ann Ford, RN, MSc, is the Associate Director of the BC Hepatitis Program, Vancouver General Hospital, Vancouver, British Columbia.

Eric M Yoshida, MD, MHSC, FRCPC, FACP, FACG, is Professor of Medicine and Head of the Division of Gastroenterology, The University of British Columbia, Vancouver, British Columbia.

Nilufar Partovi, BSc(Pharm), PharmD, is Clinical Coordinator in the Pharmacy Department, Vancouver General Hospital, Vancouver, British Columbia.

\section{Address correspondence to:}

Dr Tony K L Kiang

Department of Pharmacy

St Paul's Hospital

1081 Burrard Street

Vancouver BC V6Z 1Y6

e-mail: tkiang@gmail.com 